\title{
APT CRYOMODULE ASSEMBLY PROCESS AND MOCKUP MODEL ${ }^{*}$
}

\author{
B. Campbell, K.C.D. Chan, E. Newman \\ Los Alamos National Laboratory
}

\section{Abstract}

The cryomodule for the APT $\beta=0.64$ high energy accelerator incorporates either two or three RF cavities, two sets of magnetic shields, one active heat shield, multilayer insulation blankets, cryogenic plumbing for the shields and cavities, power couplers, cavity spokes and cavity frequency tuners. Maintaining cavity cleanliness is the most important aspect of the cryomodule assembly. The clean assembly of the cryomodule is done in a class 100 clean room. The final assembly, such as tungsten inert gas welding used to join the cryogenic plumbing, is done in a controlled environment. To address the assembly issues early in the design process, a full-scale mock-up of the entire cryomodule was fabricated. In this paper we will also describe the assembly process, and illustrate how the mock-up facilitated the procedures.

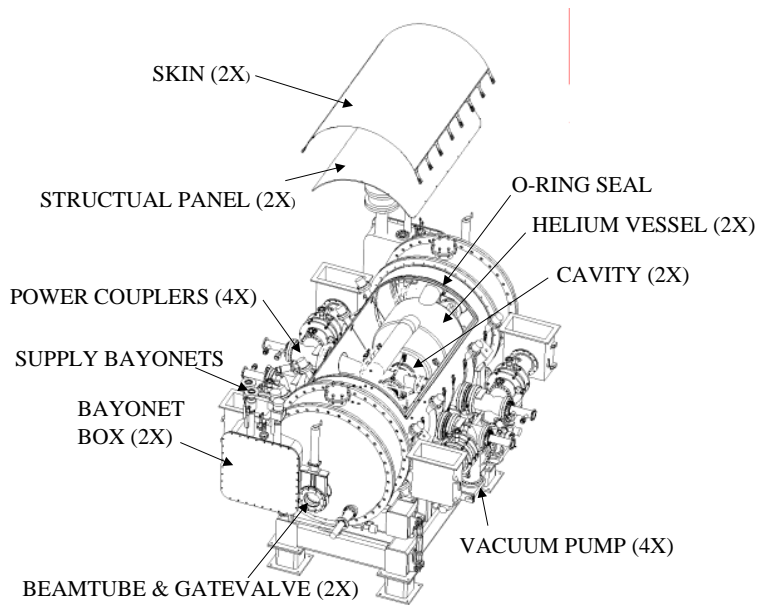

Figure 1: The $\beta=0.64$, two cavity, ED\&D cryomodule.

\section{INTRODUCTION}

When the design of the superconducting accelerator was initiated for the APT program, the cryomodule stood out as one of the important building blocks. Failure or success of the accelerator depends on how well the cryomodule performs. It is the centrepiece, where all of the components are integrated together. The program made a decision to build a mock-up of the cryomodule before the real one would be fabricated. This mock-up would allow the designers and engineers to review their designs in a full-scale model before they were implemented later.

The six requirements for this mock-up are: 1) it will represent the actual dimensions with enough detail to effectively represent the final product. 2) it will be constructed out of various materials, which are selected for ease of manufacturing and minimal cost. 3) it is a living piece of equipment, it should be capable of being revised quickly and easily. 4) it could be used, as a tool for doing planned performance experiments that otherwise couldn't be tested until the real article was built (i.e. measuring the magnetic field at the cavity). 5) the mock-up should be capable of being substituted for the real thing for fit checks and component evaluation. 6) its appearance should be satisfactory, it will be the only thing the program will have to show for a long period of time. The $\beta=0.64$ ED\&D cryomodule design is shown in Figure 1. The mock-up was modelled around this design.

\section{CRYOMODULE/MOCK-UP DESIGN}

This section will give a brief description of the current cryomodule design shown in Figure 1 and how that design is modelled in the mock-up assembly Figure 2.

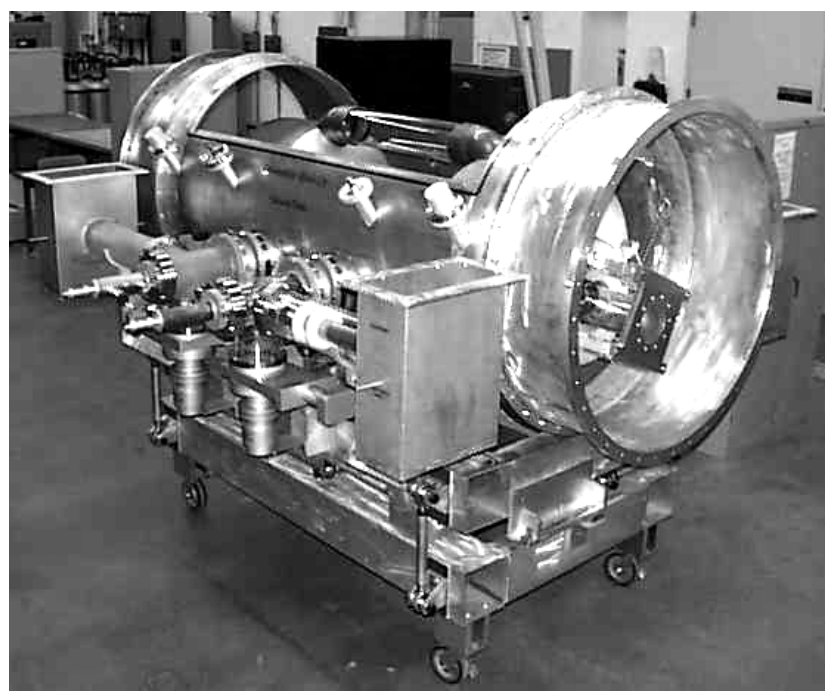

Figure 2: The $\beta=0.64$, two cavity, cryomodule mock-up.

\subsection{Vacuum Tank}

This vacuum tank uses the same design approach as was used in the CERN-LEP "wrap-up" cryomodule [1],[2]. The approach maximises accessibility to the cryomodule. The CERN-LEP wrap-up design has one fixed stave on which all of the penetrations to the cryomodule are mounted. The stave is welded at each end to the vacuum vessel end bulkheads. The vessel is sealed to the atmosphere using an elastomer o-ring. Removable staves are added around the circumference of the vessel to keep the thin cover from collapsing. A thin stainless steel cover is wrapped around the vessel covering the o-ring.

\footnotetext{
"Work supported by the US Department of Energy.

"Email: enewman@lanl.gov
} 
Buckles, with one end mounted to the cover and the other to the stave, are tightened causing the cover to compress the o-ring. This design has worked very well. There is a maximum amount of accessibility to the cavities inside when the removable staves are removed. APT adopted this approach because of the accessibility to the cavities and the success CERN has had with the design.

There are some differences between the CERN and LANL design approaches. The CERN cryomodule has one power coupler per cavity where APT will have two. The CERN power coupler penetrates the upper quadrant of the cryomodule through the only permanent stave. The two APT couplers are on opposite sides of the cavity and are on the horizontal plane. This requires the APT vessel to have 2 permanent staves. The thin stainless steel cover is split into two pieces, one on top as shown in figure 1, and one on the bottom. Two separate o-ring grooves will be machined into the vessel.

The APT cryomodule vacuum tank will have a top opening which will be approximately $120^{\circ}$ of the circular annulus. A similar large opening will be in the bottom. This provides for excellent access for assembly and laminar airflow in the clean room. This is shown in Figure 1 where the structural panel and vacuum skin are raised above and the magnetic, heat shields and the multilayer insulation are removed. This design also provides for easy cleaning of the vacuum tank before it is moved into the clean room.

The main vacuum vessel of the mock-up was fabricated out of 6061 aluminium and maintains the same material thickness and sizes as the actual design, which is 310 stainless-steel plate. It is barely tack welded together for structural integrity, and therefore is not intended to be vacuum leak tight. This represents the overall design, including the vacuum sealing techniques used on the large top and bottom openings.

\subsection{Support Stand}

The support stand is fabricated out of 8 " square 6061 aluminium tubing with 0.18 " wall thickness. The overall dimensions are the same as the real stand, but unlike the final design as the total weight of the mock-up is approximately 3,000 pounds compared to the approximate 10,000-pound weight of the real cryomodule. The mounting legs have been replaced with heavy-duty casters for ease of movement.

\subsection{Cavity and Helium Vessel}

The APT 5-cell, $\beta=0.64$ cavity is fabricated from RRR (Residual Resistivity Ratio) 250 niobium for the cavity cells and RRR 40 niobium tubing for everything else [3]. The two ends of the cavity as well as on all of the ports protruding from the beamtubes have stainless-steel conflat ${ }^{\circledR}$ flanges brazed to them. A titanium liquid helium vessel encloses the cavity to provide the $2.15 \mathrm{~K}$ environment the cavity will operate in. Each cavity assembly is supported with eight diagonal spokes from the vacuum vessel wall. The mock-up cavity assemblies are constructed out of a combination of aluminium and wood. This assembly only represents the outer contour of the helium vessel and cavity including the tuner mechanism. The eight diagonal spokes are included and are mocked-up using brass threaded rod

\subsection{Power Coupler}

The APT power couplers are designed to deliver a maximum of $250 \mathrm{~kW}$ per coupler to the cavity [4]. There are 2 couplers per cavity. A WR1500 waveguide section is transitioned to a coax line. There will be two coaxial windows in the line to make the air to vacuum transition. The coax line will go through a right angle transition into the power coupler. The power coupler is a coaxial line with the outer conductor fabricated from copper plated stainless steel. The inner conductor is fabricated from oxygen free copper. The assembly is supported from the cryomodule using a flexure, which constrains the unit in five degrees of freedom. The transverse direction is left to permit contraction during cool down.

The mock-up power coupler outer conductors are modelled from acrylic tubing. One station is clear to view the internal components. All vacuum flanges are commercially available ConFlat type knife-edge seals. The gate valves and turbo pumps are of wood.

\section{ASSEMBLY PROCEDURE}

The assembly procedure for the cryomodule is complex and important. Having the mock-up greatly visualises this entire process and allows for testing and developing these procedures. The superconducting cavity is extremely sensitive to dust and any type of contamination on its interior surface. Therefore the assembly of the cavity and its components must be done in a very clean environment. A class-100 clean room will be used to do the assembly. To minimise the number of components to be brought into the clean room, the assembly is divided into two phases. The first phase, the clean assembly, includes the components that have to be assembled in order to seal the cavity. Once sealed, contaminants cannot enter the cavity. The cavity can then be moved outside the clean room into phase 2 the final assembly. The remainder of the equipment would then be installed without concern of contaminating the cavity.

\subsection{Clean Assembly}

The assembly in the clean room would consist of the vacuum tank, 2 cavity/helium vessel assemblies, 4 power coupler assemblies, 4 HOM couplers, 2 rf pick-up probes, an inter-cavity spool piece, 16 spokes, 2 gate valves and all of the assembly hardware. This assembly has been demonstrated on the mock-up. The mock-up clearly shows that it is impossible to approach the vacuum tank from either side. The location of the power couplers in 
the APT design blocks the access to the center of the cryomodule. The mock-up shows the necessity of a tool to support the vacuum vessel and also provides access from underneath.

\subsection{Final Assembly}

The final assembly of two of magnetic shields, one active heat shield, multi-layer insulation blankets, extensive cryogenic plumbing, and remaining components are then installed outside the clean room. The power couplers do not allow access to the cryomodule during this process. A rotating fixture was developed so the cryomodule can be rotated $90^{\circ}$ to allow access to the center Figure 3 .

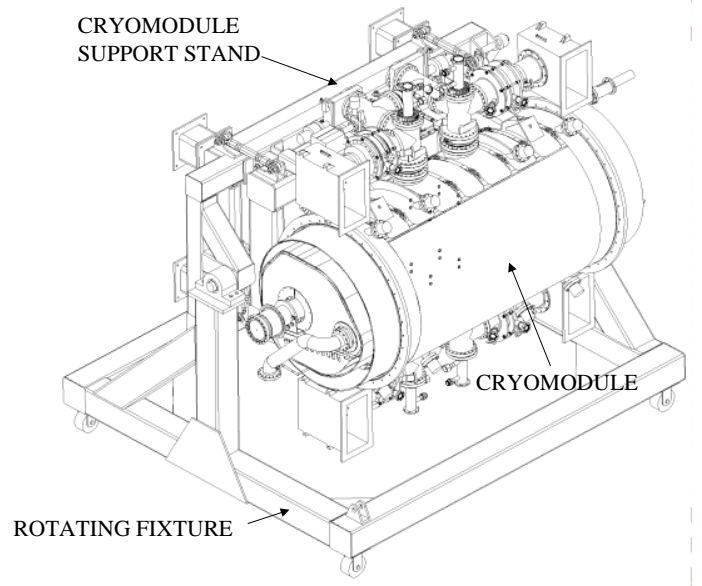

Figure 3: Rotating assembly fixture.

\section{SIX MOCK-UP REQUIREMENTS}

\subsection{Representation of the final product}

The components of the mock-up assembly were designed after reviewing the actual drawings and layouts. Where it was critical to have the actual size of the component, the mock-up component would have the same dimensional tolerances. In other areas where the dimensions weren't so critical, off-the-shelf components were used. Material selection was very important.

\subsection{Material Selection:}

The materials for the mock-up components were selected to facilitate the manufacturing process and minimise cost, but still provide an accurate model where required. Wood was used as the fabrication material in many places. It is cheap and easy to work with, it is easy to modify and it doesn't have any residual magnetic fields.

No magnetic materials were incorporated into the mock-up. The stainless-steel conflat ${ }^{\circledR}$ flanges were the only potential source of magnetic fields in the actual cavity design. The same flanges were used in the mockup. This should create an accurate assessment of the magnetic fields exposed to the cavity.

\subsection{Easy to Modify:}

The design of the mock-up allowed it to be modified easily. Since many of the components are made from wood and other cheap easy to work with materials, modifications are easy to do.

\subsection{Experimental Tool}

The mock-up will be used to evaluate the effectiveness of the magnetic shielding. This way the magnetic shielding can be evaluated for sufficiency before the cryomodule is fabricated.

\subsection{Component Evaluation}

The mock-up can give the designer and engineer time to reflect on the design. In one instance, after reviewing the cavity support system, a potential virtual leak was discovered. The gas trapped in the capsule over the spoke adjustment mechanism had no way to be evacuated.

Also the mock-up could be used to fabricate components in parallel. The thermal and magnetic shields could be fit checked on the mock-up prior to being installed in the cryomodule. The MLI thermal blankets could be trimmed using the mock-up and then installed in the cryomodule.

\subsection{Appearance}

The mock-up was available for show and tell session's two years before the actual cryomodule will be delivered. Everything in the cryomodule has been painted and looks clean and neat. It has been used as a prop to describe the concept of the superconducting accelerator.

\section{CONCLUSION}

One never appreciates the value of a mock-up until after it has been built. It is a very useful and cheap tool for evaluating ideas and designs. It is also very useful as a tool to aid others to visualise the concepts being proposed by the designers and engineers.

It helps prevent schedule delays due to redesign by highlighting shortfalls early in the fabrication cycle.

\section{REFERENCES}

[1] B.M. Campbell, "Design Status of the Cryomodules for the APT Linac", Padoua Italy, (Oct 1998)

[2] B.M. Campbell, "Engineering Design of the APT Cryomodules", for LINAC"98", Chicago, (1998)

[3] R. Gentzlinger, "Design, Analysis, and Fabrication of the APT Cavities", for PAC"99", New York City, (Mar 1999)

[4] R. E. Lujan, "Development of the SCRF Power Coupler for the APT Accelerator", for PAC"99", New York City, (Mar 1999) 